\title{
Proyecto de Hermanamiento "Improving the Skills of Forensic Experts in Turkey": un reto de futuro abierto a la Comunidad Médico Forense Española
}

\author{
Twinning Project "Improving the Skills of Forensic Experts in \\ Turkey": a challenge of future open to the Spanish Forensic \\ Medicine Community
}

En mayo de 2009 la responsable de la Secretaría de Estado de Cooperación Internacional, Da Soraya Rodríguez Ramos, impulsó la participación del Ministerio de Justicia de España en un concurso internacional para optar a la coordinación de un Twinning Project con la República de Turquía denominado "Improving the Skills of Forensic Experts". No era la primera vez que el Gobierno de España participaba en una iniciativa de este tipo, pero sí fue la primera vez en la que el Ministerio de Justicia participaba y la primera en la que para la coordinación del proyecto se contaba con la participación de miembros del Cuerpo Nacional de Médicos Forenses (CNMF).

Los Twinning Projects o Proyectos de Hermanamiento constituyen una herramienta diseñada por la Unión Europea (UE) para la adaptación a los estándares europeos de las administraciones públicas de

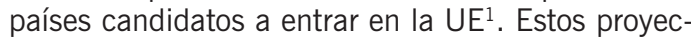
tos, financiados con fondos de la Unión Europea, desarrollan actividades formativas tanto en el país candidato como en países miembros destinadas al intercambio de información en un área de trabajo entre miembros de administraciones del país candidato y países comunitarios. Su objetivo no es tanto el desarrollo de bases formativas sino el intercambio de modelos de trabajo y experiencias profesionales con el fin de ayudar a cada nuevo futuro estado miembro a encontrar el modelo de trabajo que permita la mejora de su rendimiento respetando sus peculiaridades nacionales.

El proyecto presentado por España en mayo de 2009 fue aceptado por Turquía y la UE. Tras esta aprobación, se desarrolló un concierto de colaboración entre el Ministerio de Justicia y el de Interior de España y el Netherlands Forensic Institute de Holanda para la implementación del proyecto $^{2}$. El equipo europeo de trabajo estaba constituido por el médico forense Dr. Andrés Bedate, como Líder de Proyecto (Spanish Project Leader), el Dr. Jos Tóth, como coordinador de la participación holandesa (Junior Project Leader), y el Dr. Pedro Manuel Garamendi, también médico forense, como coordinador de los expertos europeos en Turquía (Resident Twinning Adviser o RTA). Contaban, además, con la colaboración en la gestión de los aspectos financieros de la Fundación Internacional y para Iberoamérica de Administración y Políticas Públicas (FIIAPP), organismo encargado de estas cuestiones para todos los proyectos de cooperación internacional del Gobierno de España ${ }^{3}$. En Turquía, las personas responsables de la colaboración fueron el Profesor Dr. Cengiz Haluk Ince, director del Consejo de Medicina Forense de Turquía (ATK), como líder turco de proyecto (Turkish Project Leader), la Dra. Ipek Esen Melez, como coordinadora de los expertos turcos (RTA Counterpart), y el Dr. Yalçin Büyük, vicepresidente del ATK, como responsable de la coordinación de aspectos materiales (Senior Project Officer) ${ }^{4}$.

El proyecto tuvo un largo proceso de negociación con el fin de definir con precisión los contenidos formativos a desarrollar y las metodologías más apropiadas para ello. De este modo, tras más de un año de negociación, en marzo de 2011 el proyecto se inició. En palabras de los responsables de la propia Delegación de la UE en Turquía, el citado proyecto sólo puede definirse como claramente ambicioso por el número de instituciones implicadas y la amplitud de las áreas de trabajo a desarrollar.

Las instituciones receptoras de las actividades son tres: la Policía Nacional y la Gendarmería de Turquía y el ya citado Consejo de Medicina Forense de Turquía. El objetivo prioritario se centra en el desarrollo de estrategias para mejorar las actividades de las ciencias forenses en Turquía en casos de posible vulneración de Derechos Humanos. Como objetivos complementarios se cuentan la implementación de mejoras en los sistemas de trabajo que permitan proceder a la acreditación de diversas técnicas de laboratorio según la norma ISO/IEC 17025, la elaboración de un Manual de Buenas Prácticas para la investigación del lugar de los hechos acorde con la norma ISO/IEC 17020 y la actualización en diversas áreas de las ciencias forenses de los expertos turcos ${ }^{5,6}$.
PM. Garamendi ${ }^{1}$

A. Bedate Gutiérrez ${ }^{2}$

C. Haluk Ince ${ }^{3}$

Y. Büyük ${ }^{4}$

I. Esen Melez ${ }^{5}$

\author{
${ }^{1}$ Médico Forense. \\ Resident Twinning \\ Adviser. Twinning Project \\ "Improving the Skills of \\ Forensic Experts". \\ TR 08 IB JH 01. \\ Consejo de Medicina \\ Forense. Estambul \\ (Turquía). \\ ${ }^{2}$ Médico Forense. \\ Project Leader. \\ Madrid (España). \\ ${ }^{3}$ Médico Forense. \\ Director del Consejo \\ de Medicina Forense. \\ Estambul (Turquía). \\ ${ }^{4}$ Médico Forense. \\ Vicepresidente del \\ Consejo de Medicina \\ Forense. Estambul \\ (Turquía). \\ ${ }^{5}$ Médico Forense. \\ Resident Twinning \\ Adviser Counterpart. \\ Consejo de Medicina \\ Forense. Estambul \\ (Turquía).
}

Correspondencia: Dr. Pedro Manuel Garamendi E-mail: imanolgaramendi@ gmail.com 
El plan global de trabajo incluye un total de 25 áreas de formación (Tabla 1), repartidas a lo largo de un total de 315 días de formación, 243 de ellos en las ciudades de Ankara e Istanbul y 72 en ciudades de la UE (Sevilla, Cáceres, Mérida, Barcelona, Madrid, Lisboa, Oporto y La Haya). El plan prevé la formación de un total de 820 expertos turcos en muy diversas áreas de las ciencias forenses. Para todo ello se cuenta con la inestimable colaboración de un total de 92 expertos europeos provenientes de 6 países distintos y con la colaboración muy significativa del INML de Portugal (Figura 1). La duración global del desarrollo del proyecto se extiende hasta marzo de 2013, durante un total de 24 meses.

El Proyecto de Twinning, que en estos momentos se encuentra ya en su noveno mes de desarrollo, constituye una oportunidad para España de colaborar en el escenario europeo en el desarrollo de las bases institucionales de la UE y constituye una excelente opción para afianzar las relaciones internacionales

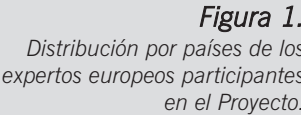

en el Proyecto.

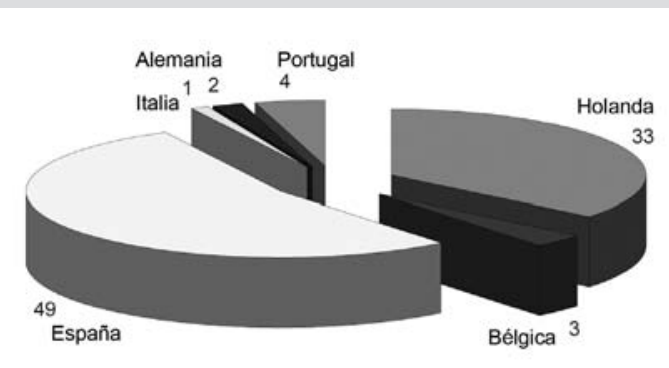

entre España, Holanda y Turquía ${ }^{7}$. Pero, además, para el CNMF es ésta una excelente oportunidad para demostrar su capacidad para asumir la planificación y ejecución de planes ambiciosos de formación sólidos y eficientes, no sólo a nivel colectivo sino también a nivel individual, merced a la colaboración en este Proyecto de múltiples médicos forenses de diversos Institutos de Medicina Legal (IML) de España que están demostrando sobradamente su calidad profesional individual y la calidad del trabajo que el colectivo forense realmente tiene en nuestro país.

Este proyecto de cooperación internacional constituye también un recordatorio a los componentes del CNMF de la necesidad de abrir sus fronteras científicas a Europa. La colaboración con Latinoamérica está ya asentada hace varios años merced a iniciativas institucionales como las del Centro de Estudios Jurídicos ${ }^{8}$, mediante la realización de cursos en las diferentes sedes de Agencia Española de Cooperación Internacional para el Desarrollo $(A E C I D)^{9}$ y la presencia de miembros del CNMF en el IML de Bogotá, en la sede del Equipo Argentino de Antropología Forense en la ciudad de Buenos Aires, en las ciudades de Lisboa y Oporto en Portugal, y más recientemente en Inglaterra ${ }^{10}$. Además, recientemente, España ostenta la Secretaría Permanente de Red de Instituciones de Medicina Legal y Ciencias Forenses de Iberoamérica, figurando como secretario de la misma el Dr. Andrés Bedate. Esta Red reúne a la totalidad de IMLs de Iberoamérica, habiéndose desarrollado en el mes de septiembre, su cuarta Asamblea General en la Ciudad de Funchal (Portugal). Sin embargo, el presente proyecto constituye el primer paso

Tabla 1.

\begin{tabular}{|c|c|c|}
\hline MEDICINA FORENSE & $\begin{array}{l}\text { LABORATORIO DE CIENCIAS } \\
\text { FORENSES Y C.S.I. }\end{array}$ & DERECHOS HUMANOS \\
\hline Antropologia Forense & Genética Forense & $\begin{array}{l}\text { Investigación forense en casos de posible } \\
\text { vulneración de Derechos Humanos }\end{array}$ \\
\hline Patología Forense & Análisis forense de drogas de abuso & \\
\hline Microbiología Forense & $\begin{array}{l}\text { Tecnologia digital (sistemas informáticos, } \\
\text { imagen digital) }\end{array}$ & \\
\hline Psiquiatria Forense & $\begin{array}{l}\text { Balistica forense externa (reconstrucción } \\
\text { de escenarios de disparos) }\end{array}$ & $\begin{array}{l}\text { NORMAS DE CALIDAD Y } \\
\text { ACREDITACIOON }\end{array}$ \\
\hline Entomologia Forense & $\begin{array}{l}\text { Balistica forense (análisis de residuos de } \\
\text { disparos) }\end{array}$ & Acreditación según norma ISO/IEC 17025 \\
\hline Odontología Forense & Reconstrucción de Accidentes de tráfico & Acreditación según norma ISO/IEC 17020 \\
\hline Estimación de edad en sujetos vivos & $\begin{array}{l}\text { Investigación de escenarios tras } \\
\text { explosiones }\end{array}$ & $\begin{array}{l}\text { Elaboración del libro de Buenas Prácticas } \\
\text { en C.S.I. }\end{array}$ \\
\hline Agresiones sexuales & Trazas instrumentales & \\
\hline Violencia de género contra las mujeres & Análisis de explosivos & \\
\hline Toxicología Postmortem & Estudio de manchas de sangre & \\
\hline $\begin{array}{l}\text { Evaluación del menor víctima de } \\
\text { agresiones (Psicología Forense) }\end{array}$ & Análisis forense de audio y voz & \\
\hline
\end{tabular}


colectivo hacia la apertura al escenario forense europeo, apertura ya realizada hace varios años por otros profesionales de las ciencias forenses (Instituto Nacional de Toxicología y Ciencias Forenses, Cuerpo Nacional de Policía, Guardia Civil), pero a la que aún el colectivo médico forense español no se había iniciado.

Sin duda, cada cosa debe suceder en su momento. No es casualidad que este inicio de apertura a Europa se haya llevado a cabo en un periodo durante el cual hemos visto la implementación y desarrollo de las estructuras de los IMLs con su orientación hacia la focalización del trabajo forense por áreas de conocimiento y hacia el asentamiento de una conciencia colectiva de la necesidad de unos criterios de calidad en el desempeño profesional. En este mismo periodo se han dado los pasos institucionales hacia la integración dentro del sistema MIR de la especialidad de medicina legal y forense, paso esencial para el desarrollo de una formación profesional de calidad de los médicos forenses merced a la integración de las ciencias forenses dentro del marco general académico y del resto de especialidades médicas, necesidad que el colectivo profesional venía demandando hace ya varios años. La reciente publicación de los volúmenes del Tratado de Medicina Legal y Ciencias Forenses dirigida por el Dr. Santiago Delgado Bueno es también el fiel reflejo de la asentada integración de los médicos forenses con otros técnicos en ciencias forenses, juristas y departamentos universitarios en el ámbito académico y de investigación ${ }^{11}$. Se han creado sociedades científicas de ámbito nacional con un claro espíritu de apertura internacional, como la
Sociedad Española de Patología Forense (SEPAF) ${ }^{12} 0$ la Asociación Española de Antropología y Odontología Forenses (AEAOF) ${ }^{13}$ y los médicos forenses españoles empiezan a ser colaboradores habituales en revistas y tratados científicos internacionales. Sin duda éste era el momento adecuado para la apertura a Europa de los médicos forenses españoles. Existe una franca inquietud entre éstos por la investigación, la orientación docente y los criterios de calidad en el ejercicio profesional y sólo en este contexto la apertura a Europa era razonable.

Este inicio de apertura, necesario para la medicina forense española, no obstante, precisa del apoyo institucional para poder asentarse. Es necesario este apoyo para el desarrollo adecuado de los medios materiales y de los currículos formativos en línea con iniciativas de harmonización y calidad, que ya son y seguirán sin duda siendo el futuro y presente de las ciencias forenses en Europa. La medicina forense española y las Administraciones Públicas españolas tienen que creer en su capacidad de dar el salto del arte a la ciencia y de asentar sus bases en un sólido sistema de trabajo que afiance definitivamente el papel de las ciencias forenses en el marco general de las disciplinas científicas. Quienes estamos implicados en este Proyecto de Hermanamiento sólo esperamos que los esfuerzos personales e institucionales invertidos en el mismo colaboren a fomentar la imagen de excelencia que la comunidad científica y médico forense española se ha ganado en estos años y que acaben redundando en beneficios palpables para la comunidad profesional.

\section{Bibliografía}

1. Sitio web de la UE sobre los Twinning Projects: http://ec.europa.eu/enlargement/how-does-itwork/technical-assistance/twinning en.htm (acceso 2/12/11)

2. Netherlands Forensic Institute (NFI). http:// www.forensicinstitute.nl/ (acceso 2/12/11).

3. Fundación Internacional e Iberoamericana para las Administraciones y Políticas Públicas. http:// www.fiiapp.org/ (acceso 2/12/11).

4. Consejo de Medicina Forense de Turquía. Adli Tip Kurumu-Council of Forensic Medicine. http:// www.atk.gov.tr/ (acceso 2/12/11).

5. ISO/IEC 17025. http://www.iso.org/iso/catalogue detail.htm?csnumber=39883 (acceso 2/12/11).

6. ISO/IEC 17020. www.iso.org/ iso/catalogue_detail? csnumber $=29342($ acceso 2/12/11)

7. Improving the skills of Forensic Experts Twinning Project. http://www.forensicskills.eu (acceso 2/12/11).

8. Centro de Estudios Jurídicos. http://www.cej-mjusticia.es/ (acceso 2/12/11).

9. Agencia Española de Cooperación Internacional para el Desarrollo. http://www.aecid.es/ (acceso 2/12/11).

10. Equipo Argentino de Antropología Forense. http://www.eaaf.org/ (acceso 2/12/11).

11. Tratado de Medicina Legal y Ciencias Forenses. Dirigido por Delgado S. Barcelona: Ed. Bosch, 2011.

12. Sociedad Española de Patología Forense. http://www.sepaf.net78.net/ (acceso 2/12/11).

13. Asociación Española de Antropología y Odontología Forenses. http://www.aeaof.com/(acceso 2/12/11). 